\title{
FACTORES DE AMPLIFICACIÓN DEL SUELO EN FUNCIÓN DEL PERIODO OBTENIDOS A PARTIR DE REGRESIONES PARA COSTA RICA
}

\author{
SOIL AMPLIFICATION FACTORS IN TERMS OF PERIOD DERIVED FROM \\ REGRESSIONS USING COSTA RICAN DATA SET
}

\author{
Víctor Schmidt
}

\author{
Laboratorio de Ingeniería Sísmica, Inst. de Investigaciones en Ingeniería, \\ Facultad de Ingeniería, Universidad de Costa Rica. \\ 2060 San Pedro de Montes de Oca, San José, Costa Rica \\ victor.schmidt@ucr.ac.cr
}

(Recibido: 29/10/2010 ; aceptado: 01/06/2011)

\begin{abstract}
Amplification factors for two soil types and 23 periods were obtained, using accelerographic records and their correlation with seismic parameters and local geology related to the site for Costa Rica. The factors were obtained from regressions between PSA (pseudo spectral acceleration) for 5\% damping as the dependent variable and three independent variables: magnitude, hypocentral distance and soil type at each site, defined as S II (hard soil) and S III (medium to soft soil). It was assumed that the condition S I (rock) does not amplify seismic waves in the range of periods defined. Factors obtained for S II shows an almost constant value throughout the range of periods for the three different data sets considered (subduction, crustal or crustal + subduction combined) and compared with amplifications obtained by other authors, especially for Japan. For S III, amplification factors obtained in this investigation for the entire data set (subduction + crustal origin) are clearly higher than those proposed by other authors for Japan, mainlyabove period of $0.4 \mathrm{~s}$.

Keywords: amplification, soils, earthquakes, inversions, Costa Rica.

RESUMEN: Se obtuvieron los factores de amplificación para dos tipos de suelo y 23 periodos, usando registros acelerográficos y su correlación con datos sismológicos y la geología local asociada al sitio, para Costa Rica. Fueron obtenidos a partir de regresiones entre el PSA (pseudo aceleración espectral) para el 5\% de amortiguamiento como variable dependiente y tres variables independientes que son: la magnitud, la distancia hipocentral y el tipo de suelo en cada emplazamiento, definido como S II (suelo firme) y S III (suelo medio a blando). Se partió del supuesto de que la condición S I (roca) no amplifica las ondas sísmicas en el rango de periodos definido. Los factores obtenidos para S II muestran un valor casi constante en todo el rango de periodos, tanto para los tres distintos grupos de datos considerados (subducción, cortical o subducción + cortical combinados), como si se comparan con amplificaciones obtenidas por otros autores, en especial para Japón. Para suelo S III, los factores obtenidos en la presente investigación para todo el conjunto de datos (subducción + cortical) son evidentemente superiores a los propuestos por otros autores para Japón, principalmente a partir de $0,4 \mathrm{~s}$.

Palabras clave: amplificación, suelos, sismos, regresiones, Costa Rica.
\end{abstract}




\section{INTRODUCCIÓN}

En Costa Rica existe una de las amenazas sísmicas más altas del mundo, por lo que es fundamental llevar a cabo en forma sistemática y continua, investigaciones orientadas a la mitigación de los efectos que traen consigo los terremotos. Por lo tanto, todo esfuerzo que se haga para mitigar estos efectos, generará un impacto muy positivo en la sociedad civil, al salvaguardarse las vidas y los bienes materiales que son escasos en países en vías de desarrollo.

En la actualidad, es del criterio de la comunidad científica a nivel mundial que el procedimiento correcto para disminuir estos efectos destructivos es a través de la prevención, por medio del desarrollo de investigaciones y de las acciones correctivas correspondientes, ya que hasta la fecha los intentos para la predicción de los sismos han sido infructuosos.

A raíz de la ocurrencia del terremoto de México en 1985, cuyo hipocentro se ubicó a más de $300 \mathrm{~km}$ de distancia de la Ciudad de México, se observaron en esta urbe una gran cantidad de daños y pérdidas de vidas humanas debido a la presencia de un fenómeno muy particular asociado a los terremotos (Sauter, 1989), poco conocido hasta ese momento y que ha sido denominado efecto de sitio o efecto local de suelo. Desde la ocurrencia de este terremoto, se han llevado a cabo numerosos estudios alrededor del mundo orientados a una mejor comprensión de ese fenómeno, debido a su gran complejidad y a sus graves consecuencias.

El efecto de sitio consiste en evidentes modificaciones que sufren las ondas sísmicas que se propagan a través de estratos del suelo, principalmente de origen volcánico, lacustre o aluvial, hasta que éstas llegan a la superficie, que es donde se asientan la mayor parte de las edificaciones y obras civiles construidas por el hombre. Estas modificaciones suelen consistir en aumentos significativos de la duración de la fase fuerte del movimiento, en amplificaciones de los valores de aceleración y en el filtrado selectivo del contenido frecuencial de la señal, generándose movimientos sísmicos en superficie con periodos predominantemente largos. Los tipos de suelo que existen en Costa Rica muestran características propicias a sufrir este fenómeno, lo que justifica su estudio en forma detallada.

No todas las estructuras y edificios reaccionan de la misma forma ante un mismo terremoto. Además, esta respuesta puede variar significativamente de un sitio a otro debido a cambios puntuales en las condiciones del suelo. Por esta razón, después de la ocurrencia de un sismo fuerte se pueden observar estructuras seriamente dañadas, junto a otras que no sufrieron daños significativos. Esto se debe a que sus características dinámicas son distintas y a que su respuesta va a depender de la magnitud de las fuerzas sísmicas inducidas. La respuesta de las estructuras a un sismo depende de los niveles de aceleración, del lapso de tiempo que dura el movimiento fuerte y del contenido frecuencial de las ondas que representan al sismo. A manera de ejemplo, estructuras bajas o rígidas de uno y dos pisos van a resonar (verán amplificada su respuesta) si son sometidas a movimientos de alta frecuencia, y estructuras de varios pisos y flexibles, entrarán en resonancia con eventos sísmicos de periodos predominantes largos. El tipo de ondas a que serán sometidas las estructuras depende en gran medida de las condiciones del suelo debajo de estas obras.

A continuación se presentan los resultados de una investigación cuyo objetivo es obtener los factores de amplificación que producen las ondas sísmicas de acuerdo con la condición del suelo predominante en la superficie y en función del periodo.

Se correlacionará una variable dependiente, representada en este caso por la pseudo aceleración espectral, con una serie de variables independientes como lo son: la magnitud del sismo, la distancia y la condición de suelo en el sitio de registro. Este último término es el que se pretende cuantificar por medio de regresiones.

Se partirá de que la roca no provoca amplificación en ninguna frecuencia, por lo que este fenómeno se estimará para condiciones de suelo. 


\section{MARCO TEÓRICO Y METODOLÓGICO}

Si se parte de la siguiente ecuación, propuesta por Boore \& Joyner (1982):

$$
Y=c_{1} e^{c_{2} M}\left[e^{c_{3} D} / D^{c_{4}}\right] e^{c_{5} S_{1}} e^{c_{6} S_{2}}
$$

El término ( ) representa la fuente, ( ) corresponde a la propagación de las ondas (atenuación por distancia) y ( ) los efectos de sitio.

Para llevar a cabo la regresión, la ecuación 1 se hace lineal aplicando logaritmos base $10 \mathrm{a}$ ambos lados y se obtiene la siguiente expresión:

$$
\log Y=c_{1}+c_{2} M+c_{3} \log D+c_{4} D+c_{5} S_{1}+c_{6} S_{2}
$$

\section{Donde:}

Y es la variable dependiente, que en este caso será la pseudo aceleración espectral (PSA) para el 5\% de amortiguamiento respecto al crítico. En vista de que hay dos posibles valores para $\mathrm{Y}$, ya que existen dos componentes horizontales de registro, se utilizará la siguiente expresión:

$$
Y=\sqrt{P S A_{l} * P S A_{T}}
$$

que corresponde a la media geométrica o geometrical mean de la aceleración espectral, según sus componentes longitudinal $\left(P S A_{L}\right)$ y transversal $\left(P S A_{T}\right)$.

Cada regresión debe hacerse para un valor de $\mathrm{Y}$ que proviene de cada frecuencia escogida (en este caso serán 23 frecuencias o periodos), de manera que se tenga un espectro de factores de amplificación lo más completo posible.

Las variables independientes de la ecuación 2 son:

- Magnitud M: es la magnitud del sismo que generó el registro y en este caso será utilizada la Mw (magnitud momento). Para la mayoría de los sismos con $\mathrm{Mw} \geq 5$, fue directamente obtenida del Global Centroid Moment Tensor (CMT, 2009). Si la magnitud era inferior o bien, no fue encontrada en la base de datos mencionada, fue necesario el uso de correlaciones, de la siguiente forma:

$$
\mathrm{Mw}=2.27+(2 / 3) \mathrm{Ms}
$$

(Okel \& Romanovicz, 1994)

Donde Ms es la magnitud obtenida a partir de ondas superficiales. Además,

$$
\begin{aligned}
& \mathrm{Ms}=-4.165+1.783 \mathrm{MD} \\
& \text { (Rojas et al., 1993) }
\end{aligned}
$$

Donde MD es la magnitud con base en la duración. Combinando estas dos ecuaciones se obtiene:

$$
\mathrm{Mw}=-0.507+1.186 \mathrm{MD}
$$

- Distancia D: es una medida de distancia, para este trabajo se considerará como la hipocentral (distancia más corta al hipocentro), debido a que es único dato disponible según las agencias consultadas. En este caso se considerará $D=\sqrt{r^{2}+r_{h}^{2}}$, donde $\mathrm{r}$ es la distancia hipocentral $\mathrm{y}$ rh es un término ficticio introducido para resolver el problema de la saturación del movimiento del suelo en el campo cercano (Dahle et al., 1995; García et al., 2005).

- Tipo del suelo S1 y S2: son variables binarias que representan la geología local del sitio y son las que se busca obtener por medio de la regresión de los acelerogramas disponibles. Las variables correspondientes al suelo operan de la siguiente forma: $\mathrm{S} 1=\mathrm{S} 2=0$ si el sitio es roca, $\mathrm{S} 1$ $=1$ y S2 $=0$ si el suelo es firme, y S1 $=0$ y S2 $=1$ si el suelo es blando.

El término $c_{4}{ }^{*} D$ de la ecuación 2 corresponde a la atenuación inelástica, debida a imperfecciones en los materiales. Tiene una contribución importante cuando la distancia $\mathrm{D}$ es grande, superior a los $200 \mathrm{Km}$ por ejemplo. Sin embargo, para la mayoría de los datos disponibles, D es inferior a esa distancia, por lo que ese término no fue considerado al hacer las regresiones, lo que permitió obtener resultados más estables (Schmidt, 2010).

Para la selección de los acelerogramas, se revisaron uno a uno todos los disponibles en Costa Rica por el Laboratorio de Ingeniería Sísmica que 
hayan sido registrados en estaciones en campo libre (estructuras de hasta dos pisos, cuya interacción con el suelo no sea significativa). En vista de que algunos registros provienen de equipo analógico, pueden existir errores en el proceso de digitalización o bien, que la onda tenga forma cuadrada (niveles muy bajos de aceleración). En otros casos, los registros pueden estar cortados o incompletos. Todo esto se analizó por medio de un programa escrito en Matlab, que permite revisar visualmente problemas evidentes en los registros.

Por otro lado, los registros fueron filtrados y su línea base fue corregida. Además, se aplicó el criterio de que la razón STA (Shot Term Average) con respecto a LTA (Long Term Average) fuera entre 3 y 5 . De esta manera se logran separar registros sísmicos de vibraciones ambientales (Atakan et al., 2004).

Respecto a la clasificación de los suelos, se utilizó el procedimiento propuesto por Zhao et al. (2006b), el cual consiste en calcular las razones espectrales de los espectros de respuesta para el $5 \%$ de amortiguamiento, para todos los sismos registrados en una misma estación. Con base en el promedio de las razones, se identifica el pico máximo (tanto en periodo como la amplitud) y se aplica el procedimiento sugerido por ese autor. En el cuadro 1 se asigna la categoría de suelos a la que corresponde cada sitio.

Donde Vs30 es una clase de promedio de velocidades de ondas de corte correspondiente a los primeros $30 \mathrm{~m}$ medidos desde la superficie (BSSC, 2003). Su expresión matemática es:

$$
V s 30=\frac{\sum_{i=1}^{N} d_{i}}{\sum_{i=1}^{N} \frac{d_{i}}{V_{s i}}}
$$

Siendo di el espesor de cada capa de suelo del perfil hasta alcanzar los $30 \mathrm{~m}$ de profundidad, Vsi la velocidad de onda cortante de cada capa i en $\mathrm{m} / \mathrm{s}$ y $\mathrm{N}$ el número de capas hasta alcanzar los $30 \mathrm{~m}$.

En caso de que en una estación en particular no se cuente con suficientes registros (al menos 5) o bien, no sea posible identificar el pico máximo, los registros aportados por esa estación no son utilizados en la inversión debido a que es vital utilizar un criterio de clasificación de suelos homogéneo para todos los casos.

Como parte del procedimiento de clasificación propuesto, se estimó también el índice:

$$
S I_{K}=\frac{2}{N} \sum_{i=1}^{N} F\left(-a b s\left[\ln \left(\mu_{i}\right)-\ln \left(\bar{\mu}_{K i}\right)\right]\right)
$$

Donde:

$\mathrm{K}$ : número de clase de sitio, desde SC I hasta SC IV

$\mathrm{N}$ : número total de periodos, que en este caso será de 20 lativa

$\mathrm{F}($ ): función de distribución normal acumu-

$\mu_{i}:$ valor $\mathrm{H} / \mathrm{V}$ de amplitud promedio para el sitio de interés, para el periodo $\mathrm{i}$

$\mu_{K i}:$ valor promedio de amplitud de $\mathrm{H} / \mathrm{V}$ para todos los sitios clase $\mathrm{K}$, obtenido del promedio entre todos los sitios de la base de datos para el periodo i

Para una estación específica, el SIK es calculado para cada clase de suelo K y este sitio será clasificado en la clase cuyo valor obtenido de SI sea máximo.

Esta clasificación fue hecha en Schmidt (2010), donde se pueden encontrar más detalles sobre la asignación del tipo de suelo a cada estación. En esa publicación se justifica que los tipos de suelo S III y S IV se agrupan como una sola categoría, debido a la insuficiencia de datos correspondientes a S IV. Entonces, los tres tipos de emplazamiento considerados serán: S I (roca), S II (suelo firme o rígido) y S III (suelo blando o muy blando).

Una vez obtenidas las variables independientes y calculadas las dependientes, se procedió a hacer las inversiones para cada una de las frecuencias seleccionadas. En este caso, se utilizó el procedimiento de regresión de mínimos cuadrados ya que fue el que presentó un comportamiento más estable para todas las frecuencias, respecto a otros como la regresión ordinaria de dos pasos (Joyner \& Boore, 1981) o el método de máxima verosimilitud (Joyner \& Boore, 1993).

Finalmente, los factores de amplificación dependientes de la frecuencia son:

1.0 para roca o S I, 10C5 para suelo tipo S II y $10 \mathrm{C} 6$ para suelo tipo S III 
Cuadro 1

Clasificación de suelos según el periodo natural del sitio, el promedio de las velocidades de onda cortante y su equivalencia según el código NEHRP (Nacional Earthquake Hazards Reduction Program, BSSC, 2003). Cuadro tomado de Zhao et al. (2006b)

\begin{tabular}{cccc}
\hline Clases de sitio & Periodo natural del sitio (s) & $\begin{array}{c}\text { Promedio de las velocidades } \\
\text { de onda cortante }(\mathrm{m} / \mathrm{s})\end{array}$ & $\begin{array}{c}\text { Equivalencia con la clasifi- } \\
\text { cación del NEHRP }\end{array}$ \\
\hline SC I (roca/suelo rígido) & $\mathrm{T}_{0}<0,2$ & $\mathrm{Vs} 30>600$ & $\mathrm{~A}+\mathrm{B}$ \\
SC II (suelo duro) & $0,2=\mathrm{T}_{0}<0,4$ & $300<\mathrm{Vs} 30=600$ & $\mathrm{C}$ \\
SC III (suelo medio) & $0,4=\mathrm{T}_{0}<0,6$ & $200<\mathrm{Vs} 30=300$ & $\mathrm{D}$ \\
SC IV (suelo blando) & $\mathrm{T}_{0} \geq 0,6$ & $\mathrm{Vs} 30=200$ & $\mathrm{E}$
\end{tabular}

Los coeficientes C5 y C6 representan los efectos del sitio según la ecuación 2.

\section{DATOS UTILIZADOS}

En esta sección se presenta la distribución de los datos usados según distintos criterios de clasificación.

En el cuadro 2 se indican los códigos de las estaciones consideradas, así como su lati- tud y longitud y el tipo de suelo asignado según Schmidt (2010). Se observa que son muy pocas las estaciones con condición de suelo S IV. Fueron consideradas 30 estaciones acelerográficas en total.

En la figura 1 se muestra la ubicación de las estaciones acelerográficas dentro del territorio nacional. Se observa que la mayoría se concentra en el Valle Central. Algunas se ubican en la Península de Nicoya, en el Pacífico Central y Sur del país y en el Caribe Central. Solamente un $10 \%$

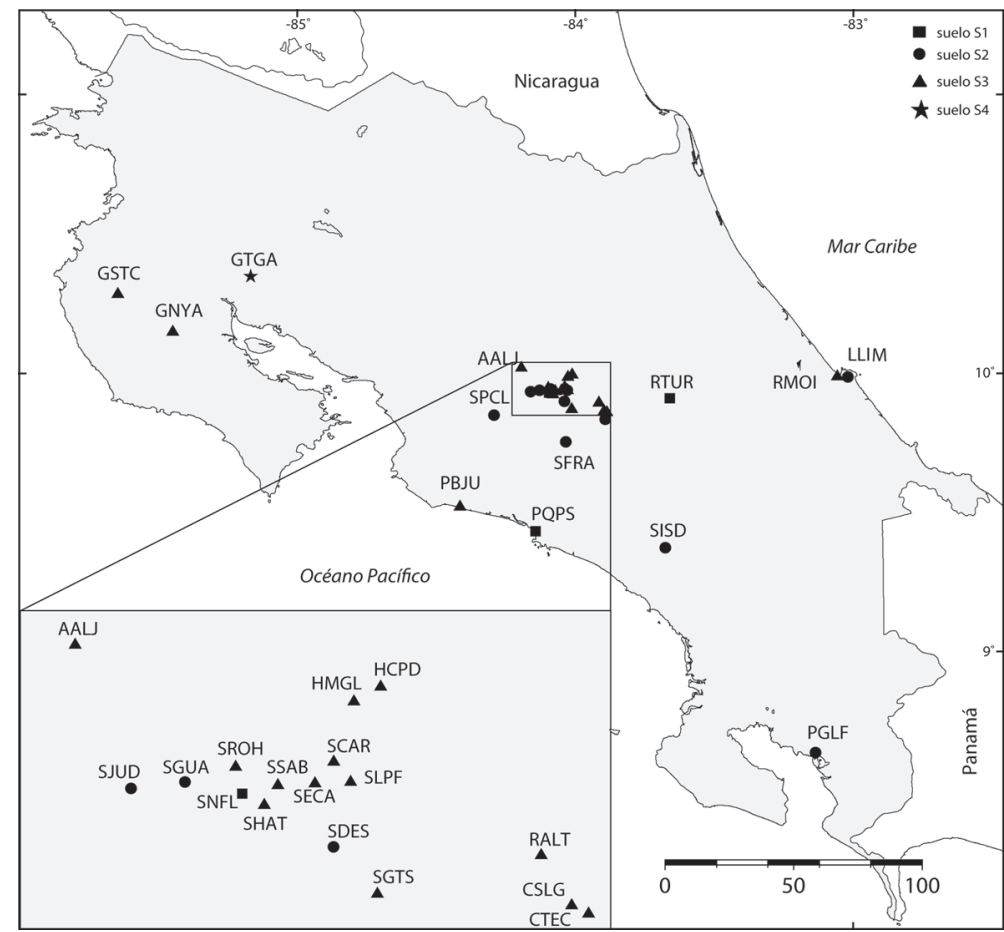

Fig. 1: Ubicación de las estaciones acelerográficas utilizadas. 
Cuadro 2

Estaciones utilizadas en el estudio según su código, coordenadas y tipo de suelo

\begin{tabular}{|c|c|c|c|}
\hline Estación & Latitud & Longitud & Suelo \\
\hline AALJ & 10,02 & $-84,217$ & S3 \\
\hline $\mathrm{CCDN}$ & 9,834 & $-83,918$ & $\mathrm{~S} 2$ \\
\hline CSLG & 9,864 & $-83,923$ & S3 \\
\hline CTEC & 9,859 & $-83,913$ & S3 \\
\hline GNYA & 10,148 & $-85,455$ & S3 \\
\hline GSTC & 10,284 & $-85,65$ & S3 \\
\hline GTGA & 10,349 & $-85,178$ & S4 \\
\hline HCPD & 9,995 & $-84,036$ & S3 \\
\hline HMGL & 9,986 & $-84,052$ & S3 \\
\hline LLIM & 9,986 & $-83,056$ & $\mathrm{~S} 2$ \\
\hline PBJU & 9,52 & $-84,435$ & S3 \\
\hline PGLF & 8,635 & $-83,171$ & $\mathrm{~S} 2$ \\
\hline PQPS & 9,432 & $-84,166$ & S1 \\
\hline RALT & 9,894 & $-83,941$ & S3 \\
\hline RMOI & 9,989 & $-83,095$ & S3 \\
\hline RTUR & 9,91 & $-83,69$ & S1 \\
\hline SCAR & 9,95 & $-84,064$ & S3 \\
\hline SDES & 9,899 & $-84,064$ & S2 \\
\hline SECA & 9,936 & $-84,097$ & S3 \\
\hline SFRA & 9,753 & $-84,058$ & $\mathrm{~S} 2$ \\
\hline SGTS & 9,871 & $-84,038$ & S3 \\
\hline SGUA & 9,938 & $-84,152$ & S2 \\
\hline SHAT & 9,924 & $-84,105$ & S3 \\
\hline SISD & 9,373 & $-83,705$ & $\mathrm{~S} 2$ \\
\hline SJUD & 9,934 & $-84,184$ & S2 \\
\hline SLPF & 9,938 & $-84,054$ & S3 \\
\hline SNFL & 9,931 & $-84,118$ & S1 \\
\hline SPCL & 9,85 & $-84,313$ & $\mathrm{~S} 2$ \\
\hline $\mathrm{SROH}$ & 9,947 & $-84,122$ & S3 \\
\hline SSAB & 9,943 & $-84,113$ & S2 \\
\hline
\end{tabular}

corresponde a suelo $\mathrm{S}$ I y más de la mitad a suelos S III y S IV, unidos como un mismo tipo debido a la poca cantidad de estaciones tipo S IV.

Con respecto a los sismos considerados, se cuenta con 349 ocurridos entre 1983 y 2009. De ellos, un $55 \%$ es clasificado según su profundidad debido a fallamiento en la placa cabalgante y un $45 \%$ por subducción, según criterios detallados en Schmidt (2010). Según estos datos, un 80,5 $\%$ tienen magnitudes $\mathrm{Mw}$ inferiores a 5, un 18,6 $\%$ a Mw entre 5 y 7 y solamente un $0,9 \%$ a Mw superior a 7,0 .

En la figura 2 se muestran los epicentros de los sismos considerados y se observa que la mayor parte se ubica en la región central del país, tanto en el Valle Central como fuera de la costa, en el Pacífico Central.

La separación entre eventos por subducción y por fallamiento en la placa cabalgante se llevó a cabo según la información reportada por cada agencia sismológica. Sin embargo, en la mayoría de los casos no existe un estudio específico al respecto, por lo que fue necesario asumir un criterio más simplificado basado en la profundidad (h), que corresponde a considerar eventos ocurridos en la placa cabalgante cuando $\mathrm{h}<25 \mathrm{~km}$ y de subducción cuando $h \geq 25 \mathrm{~km}$. Este criterio es sustentado de acuerdo a resultados obtenidos en estudios en Costa Rica (Sallares et al., 2000; DeShon et al., 2003; Warren, et al., 2008) o en observaciones obtenidas en Japón (Zhao et al., 2006a) que tiene un entorno sísmico similar a América Central.

Respecto a los registros utilizados, se contabilizaron 770 , de los cuales un $49 \%$ se asocia a sismos en la placa cabalgante y un $51 \%$ a sismos originados por subducción, lo que evidencia una equivalencia en cuanto al número de sismos según las dos profundidades consideradas.

$\mathrm{Si}$ se considera el tipo de suelo, solamente un $15 \%$ de los registros proviene de S I, un $36 \%$ de S II y un $49 \%$ de S III + S IV. Estos porcentajes son semejantes a los observados al clasificar las estaciones acelerográficas según los mismos tipos de suelo.

De acuerdo al rango de magnitudes, son pocos los registros disponibles para magnitudes $\mathrm{Mw}$ superiores a 7,0, ya que los datos se concentran en magnitudes inferiores a 5,0.

La mayor cantidad de registros disponibles fueron obtenidos a distancias hipocentrales entre 30 y $100 \mathrm{~km}$ y un bajo porcentaje a hipo-distancias superiores a $200 \mathrm{~km}$.

La clasificación de los registros puede ser observada en porcentajes en la fig. 3, según distintos criterios. Con base en esta figura, el tipo de suelo predominante es el S III, las magnitudes más 


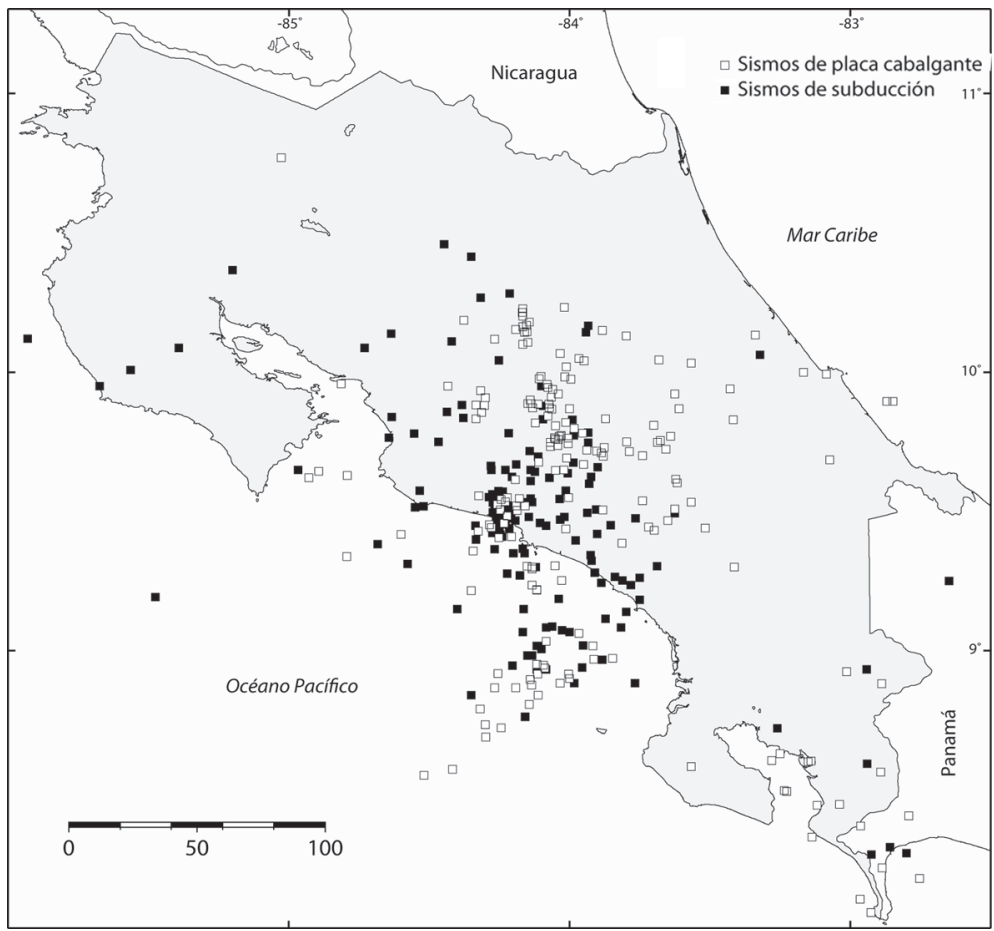

Fig. 2: Ubicación de los epicentros de los sismos utilizados en el presente estudio. Fuente: Red Sismológica Nacional.

comunes van de 4,0 a 4,9 y el rango de distancias hipocentrales en el que se concentra la mayoría de los datos va de $30 \mathrm{~km}$ a $100 \mathrm{~km}$.

El cuadro 3 muestra los factores de amplificación obtenidos para dos condiciones de suelo distintas y para 23 periodos seleccionados.

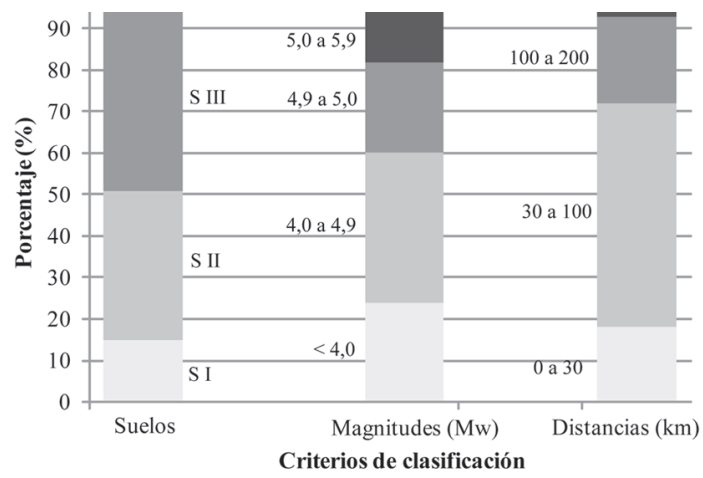

Fig. 3: Clasificación de los registros acelerográficos utilizados según: tipo de suelo, rango de magnitudes y rango de distancias hipocentrales.
Estos fueron obtenidos separando los datos según su profundidad: por subducción, por fallamiento en la placa cabalgante y combinando ambos orígenes. Estos resultados son graficados en las figs. 4 y 5 .

La figura 4 representa los coeficientes obtenidos en función del periodo para condición de suelo S II (firme). En puntos negros se muestran los factores calculados y se hacen interpolaciones entre ellos por medio de líneas rectas.

Desde $0,05 \mathrm{~s}$ hasta $0,7 \mathrm{~s}$, los coeficientes obtenidos a partir de registros cuyo sismo es asociado a fallas en la placa cabalgante son algo mayores que de los subducción y que los estimados al usar ambos orígenes combinados (toda la base de datos utilizada). Entre $0,7 \mathrm{~s}$ y $1,5 \mathrm{~s}$, las tres curvas son muy semejantes y a partir de $1,5 \mathrm{~s}$, los registros de sismos por subducción dan factores de amplificación ligeramente superiores a los otros dos casos, hasta $5,0 \mathrm{~s}$.

El caso correspondiente a sismos combinados (subducción + corticales) representa 


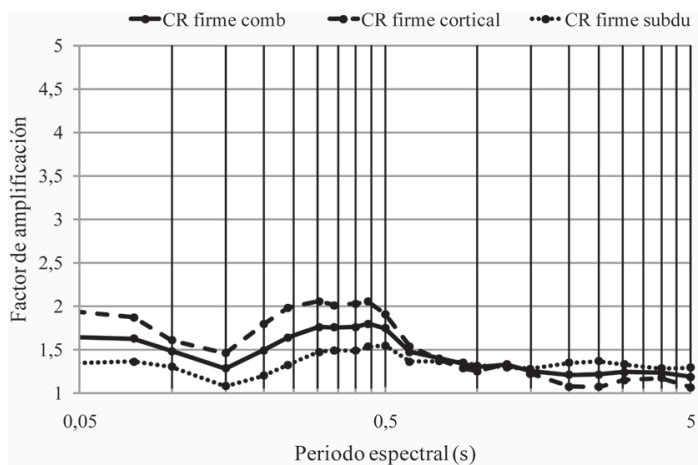

Fig. 4: Comparación entre resultados obtenidos para suelo S IIde Costa Rica separados según su origen.

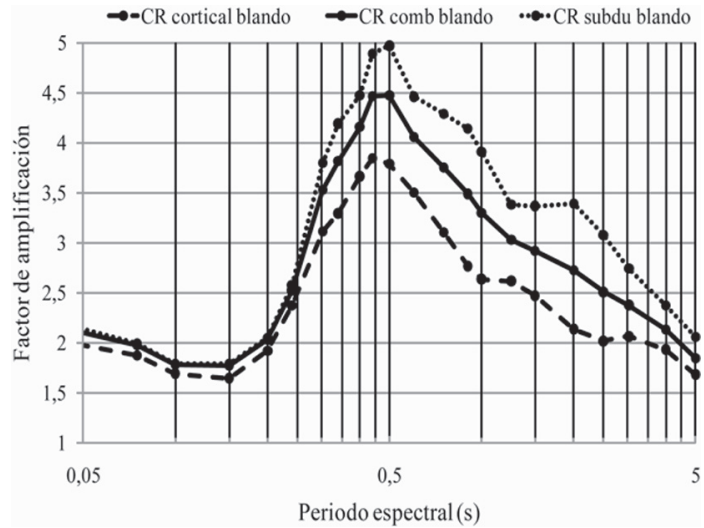

Fig. 5: Comparación entre resultados obtenidos para suelo $\mathrm{S}$ III para Costa Rica separados según su origen.

aproximadamente un promedio con respecto a considerar cada caso por separado, aún cuando las diferencias observadas no son muy grandes.

La figura 5 compara los tres grupos de coeficientes obtenidos para la condición de suelo S III (medio a blando). En este caso, se observan diferencias más notables a partir de $0,24 \mathrm{~s}$, siendo los datos por subducción los que generan factores de amplificación mayores. Esto puede estar asociado al hecho de que este tipo de sismos seleccionados para este estudio tienen en general, magnitudes más grandes que los corticales, lo que implica que los primeros tienen un contenido frecuencial más amplio. Los sismos de origen en la placa cabalgante por su parte, al tener magnitudes menores tienen un predominio de frecuencias altas.

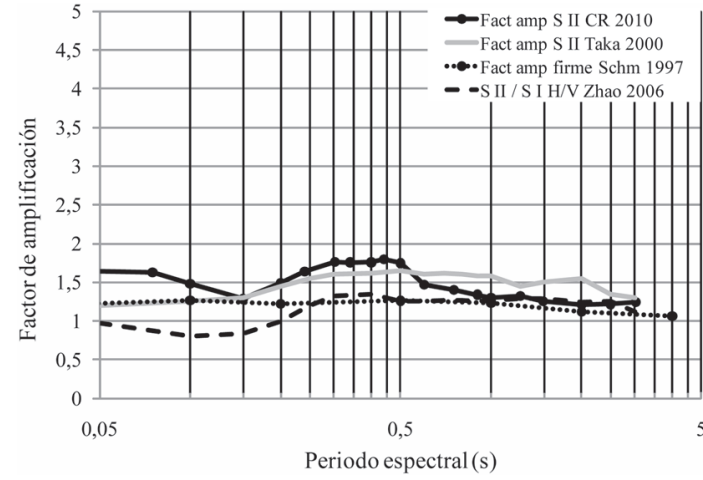

Fig. 6: Comparación entre resultados obtenidos para suelo S II, según el presente estudio (CR), Zhao et al. (2006b) y Takahashi et al. (2000).

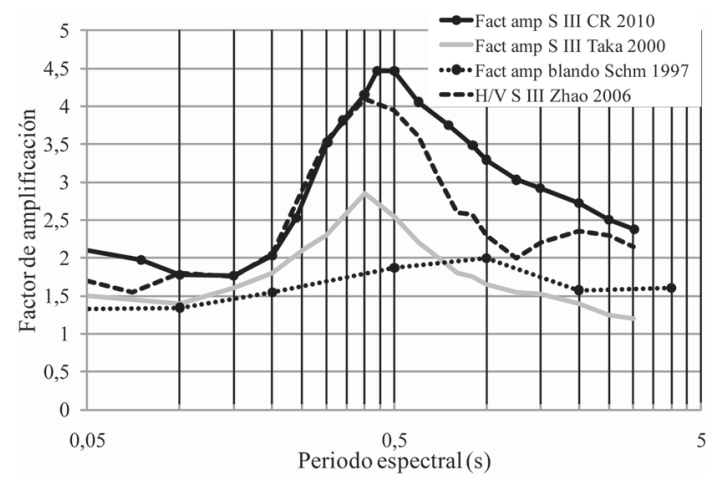

Fig. 6: Comparación entre resultados obtenidos para suelo S II, según el presente estudio (CR), Zhao et al. (2006b) y Takahashi et al. (2000).

Por otro lado, la distancia hipocentral tiene influencia en el contenido frecuencial de la señal, en el sentido de que para distancias largas, las altas frecuencias son filtradas en mayor medida que para distancias cortas. En general, los sismos de subducción tienen distancias hipocentrales mayores que los de fallamiento en la placa cabalgante y por lo tanto, son filtrados en las altas frecuencias.

Debido a lo anterior, es de esperarse que a partir de periodos bajos (altas frecuencias), los factores de amplificación se separen, siendo mayores los obtenidos con datos por subducción, ya que estos tienen un contenido frecuencial predominante en bajas frecuencias (periodos largos).

Se observa además que semejante a lo sucedido con el caso de suelo S II, para S III la curva obtenida a partir de todo el grupo de datos 
representa una especie de promedio de estos dos casos (subducción y fallamiento en la placa cabalgante) considerados por separado, además de que muestra una tendencia más suavizada. Entonces, para comparar los resultados de esta investigación con los obtenidos por otros autores, se seleccionaron las estimaciones hechas a partir de todo el grupo de datos, ya que representan una especie de promedio.

En la figura 6 se comparan los factores de amplificación para suelo S II para Costa Rica según el presente estudio (línea negra continua) con los obtenidos según Takahashi et al. (2000) (línea gris), Schmidt et al. (1997) (línea de puntos) y con la razón espectral S II/S I según Zhao et al. (2006b) (línea negra discontinua), que representa los pseudo factores de amplificación obtenidos según Zhao et al. (2004).

Se observa una gran similitud entre las cuatro trazas, siendo ligeramente inferior la que corresponde a la razón espectral H/V para Japón. Sin embargo, las obtenidas a través de inversiones con datos de Costa Rica (presente estudio) y Japón (Takahashi et al., 2000) son muy semejantes.

Al hacer las comparaciones con los factores obtenidos por Schmidt et al. (1997), que se obtuvieron con datos registrados en Costa Rica hasta esa fecha, se observa que los propuestos por ese estudio son casi constantes para todo el tango de periodos (factor de amplificación cercano a 1,2 en casi todos los casos), aunque fueron calculados para pocos periodos. Sin embargo, muestran una tendencia semejante respecto a los demás casos considerados, para suelo firme (S II).

Se realizó la misma comparación para suelo S III (medio a blando) (Fig. 7), en la que la línea negra continua representa los factores de amplificación para Costa Rica obtenidos en esta investigación, la línea discontinua corresponde a la razón espectral $\mathrm{H} / \mathrm{V}$ para suelo $\mathrm{S}$ III según Zhao et al. (2006b) y la línea gris continua muestra las amplificaciones según los modelos de atenuación propuestos por Takahashi et al. (2000), usando la clasificación de suelos propuesta por Zhao et al. (2006b) y la línea de puntos los factores de amplificación obtenidos por Schmidt et al. (1997), para datos de Costa Rica registrados hasta esa fecha.
Al comparar los factores obtenidos en este estudio con las razones espectrales H/V para Japón, se observa una gran similitud entre ambas curvas desde $0,05 \mathrm{~s}$ hasta $0,4 \mathrm{~s}$, lo que corresponde a altas frecuencias, pero a partir de $0,04 \mathrm{~s}$, la curva obtenida para Costa Rica es superior a la propuesta por Zhao et al. (2006b) hasta 3,0 s.

Si se comparan los resultados de este estudio con los obtenidos a partir de inversiones según Takahashi et al. (2000), se observa que las estimaciones hechas para Costa Rica son mayores en todo el rango de periodos, pero esta situación se vuelve más evidente a partir de un periodo de $0,7 \mathrm{~s}$, donde las ordenadas espectrales de Costa Rica superan a las obtenidas por Takahashi et al. (2000) en un $50 \%$ aproximadamente.

Esto puede estar asociado a dos situaciones:

1- La base de datos usada por Takahashi et al. (2000) cubre un rango de magnitudes mayor, sobretodo de magnitudes altas con respecto al caso de Costa Rica. En este último caso predominan sismos con magnitudes pequeñas y moderadas (inferiores a 5,0 Mw), registrados a distancias cortas e intermedias (un $72 \%$ de los datos corresponden a distancias hipocentrales entre 0 y 100 $\mathrm{Km}$ ), por lo que los efectos de sitio parecen volverse significativos en periodos largos.

2- Tanto en América Central, como específicamente en Costa Rica, las rocas predominantes son jóvenes, principalmente del Cenozoico (Bundschuh \& Alvarado, 2007), mientras que en Japón predominan rocas del Mesozoico (Geological Survey of Japan, 2010), por lo que afloran rocas más competentes. Esta situación puede provocar las altas amplificaciones evidenciadas en ciertos periodos para Costa Rica, asociadas a rocas menos competentes.

Sin embargo, es notable la semejanza de la tendencia entre las curvas que representan los factores de amplificación obtenidos para ambas regiones, ya que ambas se incrementan a partir de $0,15 \mathrm{~s}$, llegan a un máximo en $0,4 \mathrm{~s}$ y decrecen paralelas hasta llegar a 3,0 s de periodo.

Al comparar los resultados obtenidos en este estudio con los propuestos por Schmidt et al. (1997) para suelo S III, es evidente que a pesar de que en ambos casos los datos fueron registrados en Costa Rica, los factores de amplificación 
obtenidos en el presente estudio son superiores en todo el rango de periodos. Esta diferencia puede fundamentarse en las siguientes razones:

- Los datos utilizados en 1997 tienen una menor resolución ya que corresponden a registros analógicos obtenidos hasta ese año. Los usados en el presente estudio son digitales en la mayoría de los casos y obtenidos principalmente entre los años 2000 y 2010, por lo que no se está hablando de la misma base de datos.

- La clasificación de suelos usada en 1997 corresponde a observaciones en el sitio o a interpretaciones de mapas, lo que proporciona información de capas muy superficiales, por lo tanto puede ser considerada imprecisa y en algunos casos, errónea. La utilizada en el presente estudio, obedece a un criterio de clasificación más sistemático y homogéneo, basado en varios criterios como índices de clasificación y razones espectrales H/V, según Schmidt (2010).

- Según Schmidt et al. (1997), los valores de amplificación fueron definidos con base en Boore et al. (1993) y Boore et al. (1997), debido a que el tipo de regresión bayesiana utilizada en ese estudio requirió limitar el valor de los coeficientes a un rango de variación definido para poder llevar a cabo las inversiones. Según los autores mencionados, los rangos fueron obtenidos según estudios hechos para el oeste de Norteamérica, que no corresponde en forma directa a la región en estudio. Además, los factores propuestos por Schmidt et al. (1997) fueron estimados solamente para seis periodos bastante separados el uno del otro, por lo que no es posible observar posibles variaciones entre estos valores.

\section{CONCLUSIONES}

Se obtuvieron los factores de amplificación para dos tipos de suelo y 23 periodos (frecuencias), usando registros acelerográficos y datos sismológicos de Costa Rica.

Fueron obtenidos a partir de regresiones entre el PSA (pseudo aceleración espectral) para el 5\% de amortiguamiento como variable dependiente y tres variables independientes que son: la magnitud, la distancia hipocentral y el tipo de suelo en cada emplazamiento, definido como S II (suelo firme) y
S III (suelo medio a blando). Se partió del supuesto de que la condición S I (roca) no amplifica las ondas sísmicas en el rango de periodos definido.

La clasificación de suelos adoptada se sustenta en Schmidt (2010) y es equivalente a la propuesta por NEHRP, de la siguiente forma (Zhao et al., 2006b):

- S I corresponde a suelos $(\mathrm{A}+\mathrm{B})$ según NEHRP.

- S II corresponde a C según NEHRP.

- S III equivale a D según NEHRP.

- S IV equivale a $\mathrm{E}$ según NEHRP, aunque esta categoría se unió a la S III por la poca cantidad de datos disponibles correspondientes a S IV.

La distribución de los datos utilizados se puede resumir de la siguiente forma:

- Estaciones (emplazamientos): muy pocas correspondientes a S I y muchas a S III. La mayor parte de los sitios se ubican en el Valle Central de Costa Rica.

- Sismos: de acuerdo con su profundidad (subducción o cortical), se obtuvo una distribución casi homogénea. Sin embargo, con respecto a las magnitudes, la mayor parte corresponde a $\mathrm{Mw} \leq 5,0$ y muy pocos con $\mathrm{Mw}>7,0$.

- Registros acelerográficos: son pocos los obtenidos en condición de sitio S I. Respecto a las magnitudes, la mayor parte de los datos corresponde a $\mathrm{Mw} \leq 5,0$. De acuerdo con las distancias hipocentrales, la mayor parte de los datos corresponde a distancias cortas e intermedias (entre $30 \mathrm{~km}$ y $100 \mathrm{~km}$ ).

A partir de los resultados obtenidos, los factores de amplificación para S II muestran un valor bastante semejante en todo el rango de periodos, variando entre 1,19 y 1,65 para el caso correspondiente a todo el grupo de datos combinado (usando los registros de sismos por subducción y fallamiento en la placa cabalgante), siendo un poco mayores para periodos bajos (entre $0,24 \mathrm{~s}$ y $0,5 \mathrm{~s}$ ).

Los valores más altos obtenidos corresponden a los datos provenientes de sismos por fallamiento en la placa cabalgante, con factores de amplificación de 2 entre 0,24 s y 0,44 s.

Con respecto a los factores obtenidos para suelo S III, se observan diferencias notables para los tres casos considerados (registros por subducción, por fallamiento en la placa cabalgante, o por la combinación de ambos) a partir de $0,24 \mathrm{~s}$, 
obteniéndose factores de amplificación mayores para sismos por subducción, debido a que estos tienen en general magnitudes más altas y por lo tanto, un contenido frecuencial mayor que los corticales. Por otro lado, los registros debidos a sismos por subducción tienen asociadas distancias hipicentrales grandes, por lo que se encuentran filtrados en altas frecuencias y por lo tanto, muestran amplificaciones significativas en las frecuencias bajas.

Al comparar los resultados obtenidos en esta investigación para todo el conjunto de datos (subducción + fallamiento en la placa cabalgante) con los propuestos por otros autores, se observa que para suelo S II los valores de amplificación son muy semejantes en todo el rango de periodos. Sin embargo, al hacer las comparaciones para suelo $\mathrm{S}$ III, se evidencia una gran similitud con los factores obtenidos a partir de la razón H/V para Japón hasta $0,4 \mathrm{~s}$ (frecuencias altas), pero las amplitudes del presente estudio superan a las de Japón en los periodos largos (bajas frecuencias).

Esta gran amplificación mostrada por los factores obtenidos para Costa Rica se vuelve más evidente si se compara con el caso de amplificaciones obtenidas a partir de modelos de atenuación para Japón en todo el rango de periodos, debido probablemente a las distintas condiciones de suelo que predominan en ambos países, siendo los suelos predominantes en Costa Rica más jóvenes, menos consolidados y por lo tanto, más susceptibles al fenómeno de amplificación.

Sin embargo, es notable la semejanza de la tendencia entre las curvas que representan los factores de amplificación obtenidos para ambas regiones para suelo S III, ya que ambas se incrementan a partir de 0,15 s, llegan a un máximo en $0,4 \mathrm{~s}$ y decrecen paralelas hasta llegar a $3 \mathrm{~s}$ de periodo.

\section{REFERENCIAS}

ATAKAN, K., BARD, P-Y., KIND, F., MORENO, B., ROQUETTE, P, TENTO, A. \& THE SESAME TEAM, 2004: A standardized software solution for the $\mathrm{H} / \mathrm{V}$ spectral ratio technique.- $13^{\text {th }}$ World Conf. on Earth. Eng. Vancouver, Canada, paper No. 2270.
BOORE, D.M. \& JOYNER, W.B., 1982: The empirical prediction of ground motion. -Bull. Seism. Soc. Am., 81:1057-1080.

BOORE, D.M., JOYNER, W.B. \& FUMAL, T.E., 1993: Estimation of response spectra and peak accelerations from western North American earthquakes.- 72 págs. USGS Open File Report 93-509 [Internal report].

BOORE, D.M., JOYNER,W.B. \& FUMAL, T.E., 1997: Equations for estimating horizontal response spectral and peak acceleration for western North American earthquakes: A summary of recent work.- Seism. Res. Lett., 68: 128-151.

BSSC, BUILDING SEISMIC SOCIETY COUNCIL, 2003: THE 2003 NEHRP Recommended Provisions for New Buildings and Other Structures, Part 1 (Provisions) and Part II (Commentary).- FEMA 368/369. 2003 Edition. Washington D. C.

CLIMENT, A., SCHMIDT, V., HERNANDEZ, D., CEPEDA, J., CAMACHO, E., ESCOBAR, R. \& STRAUCH, W., 2007: Strong motion monitoring.- En: BUNDSCHUH, J \& ALVARADO, G. (ed.): Central America: Geology, Resources and Hazards. Taylor \& Francis. The Netherlands. Chapter, 37: 1129-1153.

CMT, 2009: THE GLOBAL-CENTROIDMOMENT-TENSOR. www.globalcmt. org. [Consulta: 10/05/2009].

DAHLE, A., CLIMET, A., TAYLOR, W. \& BUNGUM, H., 1995: New spectral strong motion attenuation models for Central America. Proceedings of the $5^{\text {th }}$ International Conference on Seismic Zonation, Vol II:1005-1012.

DESHON, H., SCHWARTZ, S., BILEK, S., DORMAN, L., GONZALEZ, V., PROTTI, J., FLUEH, E. \& DIXON, T., 2003: Seismogenic zone structure of the 
southern Middle America Trench, Costa Rica. JGR, Vol. 108, No. B10, 2491. doi:10.1029/2002JB002294.

GARCÍA, D., SINGH, S. HERRÁIZ, M., ORDAZ, M., \& PACHECO, J., 2005: Inslab earthquakes of Central México: peak ground-motion parameters and response spectra.- Bull. Seism. Soc. Am. 95: 2272-2282

GEOLOGICAL SURVEY OF JAPAN, 2010: http://www.gsj.jp/geomap/J-geology/JgeologyE.html.[Consulta: 30/10/2010].

JOYNER, W. B. \& BOORE, D. M., 1981: Peak horizontal acceleration and velocity from strong-motion records including records from the 1979 Imperial Valley, California earthquake.- Bull. Seism. Soc. Am. 71: 2011-2038.

JOYNER, W. B. \& BOORE, D. M., 1993: Methods for regression analysis of strongmotion data.- Bull. Seism. Soc. Am. 83: 469-487.

OKEL, E.A. \& ROMANOWICZ, B.A., 1994: On the variation of $b$-values with earthquake size.- Phys. Eath Planet. Inter. 87: 55-76.

ROJAS, W., BUNGUM, H. \& LINDHOLM, C., 1993: A catalog of historical and recent earthquakes in Central America.- 77 págs. Project Reduction of Natural Disasters in Central America, NORSAR, Norway [Reporte técnico No. 2-7].

SALLARES, V., DAÑOBEITIA, J. J. \& FLUEH, E. R., 2000: Seismic Tomography with local earthquakes in Costa Rica.Tectonophysics 329: 61-78.

SAUTER, F., 1989: Introducción a la Sismología.- 271 págs. Ed. Tecnológica de Costa Rica, Cartago.
SCHMIDT, V., DAHLE, A. \& BUNGUM, H., 1997: Costa Rican spectral strong motion attenuation.- 45 págs. NORSAR, Norway [Reporte técnico].

SCHMIDT, V., 2010: Avances para estudios del riesgo sísmico a escala regional y local: aplicación a América Central y a la Bahía de Cádiz (sur de España).- 307 págs. Univ. Politécnica de Cataluña, Barcelona [Tésis Ph.D].

TAKAHASHI, T., KOBAYASHI, S. FUKUSHIMA, Y., XHAO, J.X., NAKAMURA, H. \& SOMERVILLE, P.G., 2000: A spectral attenuation model for Japan using strong motion data base. 6th International Conference on Seismic Zonation, 2000. Palm Springs Riviera Resort, California: 12-15.

WARREN, L. M., LANGSTAFF, M.A. \& SILVER, P.G., 2008: Fault plane orientation of intermediate-depth earthquakes in the Middle America Trench.- J. Geophys. Res. 113. B01304. DOI: 10.1029/2007JB0050028.

ZHAO, J. X., IRIKURA, K., ZHANG, J., FUKUSHIMA, Y., SOMERVILLE, P.G., SAIKI, T., OKADA, H. \& TAKAHASHI, T., 2004: Site classification for strong-motion stations in Japan using $\mathrm{H} / \mathrm{V}$ response spectral ration.- $13^{\text {th }}$ World Conference of Earthquake Engineering, Vancouver, Canada: 1278.

ZHAO, J., ZHANG, J., ASANO, A., OHNO, Y., OOCHI, T., TAKAHASHI, T., OGAWA, H., IRIKURA, K., THIO, H.K., SOMERVILLE, P., FUKUSHIMA, YA \& FUKUSHIMA, YO., 2006a: Attenuation relations of strong ground motion in Japan using site classification based on predominant period.- BSSA, 96(3): 898-913. 
ZHAO, JOHN X., IRIKURA, K., ZHANG, J., FUKOSHIMA, Y., SOMERVILLE, P.G., ASANO, A., OHNO, Y., OOUCHI, T., TAKAHASHI, T. \& H. OGAWA, 2006b:
An empirical site-classification method for strong-motion in Japan using H/V response spectral ratio.- Bull. Sesism. Soc. Am. 96: 914-925. 